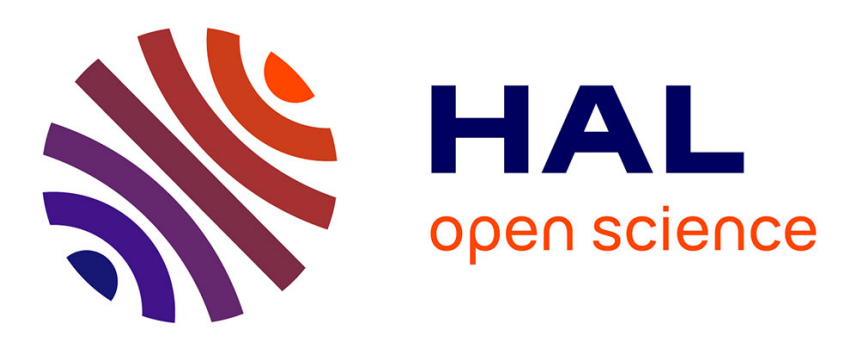

\title{
Sparse Decomposition of the GPR Useful Signal from Hyperbola Dictionary
}

Guillaume Terrasse, Jean-Marie Nicolas, Emmanuel Trouvé, Émeline Drouet

\section{To cite this version:}

Guillaume Terrasse, Jean-Marie Nicolas, Emmanuel Trouvé, Émeline Drouet. Sparse Decomposition of the GPR Useful Signal from Hyperbola Dictionary. EUSIPCO, Aug 2016, Budapest, Hungary. hal-01351242

\section{HAL Id: hal-01351242 https://hal.science/hal-01351242}

Submitted on 3 Aug 2016

HAL is a multi-disciplinary open access archive for the deposit and dissemination of scientific research documents, whether they are published or not. The documents may come from teaching and research institutions in France or abroad, or from public or private research centers.
L'archive ouverte pluridisciplinaire $\mathbf{H A L}$, est destinée au dépôt et à la diffusion de documents scientifiques de niveau recherche, publiés ou non, émanant des établissements d'enseignement et de recherche français ou étrangers, des laboratoires publics ou privés. 


\title{
Sparse Decomposition of the GPR Useful Signal from Hyperbola Dictionary
}

\author{
Guillaume Terrasse*, Jean-Marie Nicolas*, Emmanuel Trouvé ${ }^{*}$ and Émeline Drouet ${ }^{\ddagger}$ \\ * LTCI, CNRS, Télécom ParisTech, Université Paris-Saclay, 75013, Paris, France. \\ $\dagger$ LISTIC, Polytech Annecy Chambéry, Université Savoie Mont-Blanc, 74944, Annecy-le-Vieux, France. \\ $\ddagger$ CRIGEN, ENGIE, Direction Recherche et Innovation, 93211, Saint-Denis La Plaine, France. \\ (corresponding author, e-mail : guillaume.terrasse@telecom-paristech.fr)
}

\begin{abstract}
In order to improve asset knowledge and avoid third part damages during road works, the localization of gas pipes in a non-destructive way has become a wide domain of research during these last years. The Ground Penetrating Radar (GPR) is used to detect buried gas pipes. However it does not directly provide a 3D position but a reflection map also called B-scan that the user must interpret. In order to facilitate the B-scan interpretation, we propose to use a dictionary of theoretical pipe signatures. One of the most popular method to compute the coefficients is the sparse coding. Nevertheless, clutter which is noticeable by its horizontal shape makes difficult to decompose it into sparse coefficients with this dictionary. Then a low-rank matrix constraint which models the clutter is applied in order to decompose the useful signal into sparse coefficients in a blind source separation framework. Our method has been applied to simulated and real data acquired on a test area. The proposed method presents satisfying qualitative and quantitative results. Index Terms-Gas pipes localization, GPR, Dictionary, Convolutional sparse coding, Nuclear norm, Blind source separation
\end{abstract}

\section{INTRODUCTION}

While roadworks and civil engineering works operate near network pipes, an inaccurate mapping of pipe networks can cause damages which lead to human and economical damages. Precise location of buried gas networks in a non-destructive manner becomes primordial for all network system operators. In our work, we are interested in using the Ground Penetrating Radar (GPR) technology to get the position of the gas pipes. However this sensor does not provide a position in the three dimensions but a reflection map called B-scan which offers a high degree of freedom in result interpretation. Operators need experience to understand this particular data. In order to help the non-expert users of GPR to detect and find the position of buried pipes we need a tool to enhance the signal from the reflections on pipes. The ground penetrating radar has been widely used in different applications like civil engineering, geological study or glaciology [1]. This device is equipped with a transmitting and receiving antenna with a large band and a high frequency (from $100 \mathrm{MHz}$ to $2 \mathrm{GHz}$ ) placed few centimetres above soil surface. An electromagnetic wave is sent in the underground which is backscattered when it encounters a heterogeneity. The shape of the impulse signal looks like a Ricker wavelet. Then the receiving antenna records the backscattered wave at each position as function of time (vertical axis $(t)$ ). This function is called an A-scan.
By moving the GPR, a collection of A-scans is recorded at different positions known (horizontal axis $(x)$ ) thanks to an odometer. This ensemble of A-scans forms a B-scan in which each value corresponds to the amplitude of the backscattered wave at a certain position of recording and time. On a B-scan, the shape of the ensemble of echoes and their intensity inform about the nature of the object. Thereby, an hyperbolic shape means there is a point object, for instance a pipe or a rock. On the other hand, a linear shape could be a layer boundary. Therefore, to localize the pipes, we have to find hyperbolas in the B-scan. But the many backscattered waves recorded by the receiving antenna make the detection difficult.

In order to define the problem, let $Y$ be an observed B-scan which is assumed to be linearly composed of independent items: $s$ the useful signal; it results of all the echoes on pipes or layer boundaries, $c l$ clutter; it is caused by the coupling between the antenna and the soil surface. It is recognized by its horizontal shape. And $n$, noise modelled as a random process realization. In a simplified formulation, $Y$ is expressed as :

$$
Y(x, t)=s(x, t)+c l(x, t)+n(x, t)
$$

Several studies addressed the problem of removing clutter and noise in GPR data. Two distinct types of methods have been studied to remove the clutter but both of us exploit its horizontal shape characteristic. The first one models the clutter and subtracts it from the B-scan. The simplest method computes an averaged A-scan which is subtracted for each A-scan of the B-scan [1]. Other more sophisticated methods model the clutter by a linear combination of exponential functions [2]. The second kind of methods employs filters to remove clutter. The filter is computed in different domain, in the frequency-wavenumber domain [3] or in curvelet domain [4] for example. The drawback of both types of methods is their impact on the hyperbola energy.

In order to denoise seismic data a new frame with hyperbolic shape has been developed by [5]. The frame is built on three parameters. Both parameters jointly modified the thickness and shape of hyperbola. The last one influence the flattening of the hyperbola top. These parameters also act on the position of the hyperbola top. Our work has been inspired by the frame of [5] to build a dictionary of theoretical pipe signatures. Nevertheless, in our case, the atoms remain centred regardless the parameter values. 
The thickness, the shape and the flattening of the atom are controlled by independent parameters. The close atom shape with the B-scan items are exploited.

Dictionary are very popular in the signal and image processing community. They are used for denoising [6], pattern recognition [7] or blind source separation [8] applications. However, because of the dictionary dimension is much more important than the B-scan dimension, its use sets the coefficient computation and reconstruction problem. A solution consist in imposing a sparsity constraint. For example Greedy algorithms as Matching Pursuit [9] or proximal methods as FISTA algorithm [10] have been proposed.

In the subsequent part, the proposed dictionary is presented. Then the proposed method is detailed. On one hand, we show the reason that lead us to use an additional constraint with the sparsity. On the other hand, the method and the algorithm steps are explained. Finally, in order to assess the method performances, qualitative and quantitative results on simulated and real data are shown.

\section{DICTIONARY}

The dictionary is composed of hyperbolas $(g(x))$ which are convolved with a ricker wavelet $(r(t))$ according to vertical axis. Each atom is parametrized by $k=\left\{\alpha, \sigma, f, a, x_{0}, t_{0}\right\}$ We define an atom $h_{k}(x, t)$ of the dictionary :

$$
\begin{aligned}
h_{k}(x, t) & =e^{-\alpha \cdot A(x, t)} \cdot(r(t) \star \delta(t-g(x))) \\
r(t \mid \sigma) & =\frac{2}{\sqrt{3 \sigma} \cdot \pi^{1 / 4}} \cdot\left(1-\frac{t^{2}}{\sigma^{2}}\right) \cdot e^{-\frac{t^{2}}{2 \sigma^{2}}} \\
g\left(x \mid x_{0}, t_{0}, a, f\right) & =a \cdot \sqrt{f^{2}+\left(x-x_{0}\right)^{2}}+t_{0}-f \cdot a
\end{aligned}
$$

Where $\delta($.$) is the Dirac distribution. \alpha$ and function $A(x, t)$ impact the atom energy attenuation. Especially, as we are working in a finite space, $A$ ensures a low energy of the atom close to the boundaries. Parameter $\sigma$ is the standarddeviation of the Ricker wavelet and controls the thickness of the atom. Parameter $a$ affects the opening of hyperbolas (spacing of the hyperbola branches) which is directly related to the wave velocity in the soil. Then $f$ also affects the shape of the hyperbola but plays a role in the flattening of the hyperbola top located at $\left(x_{0}, t_{0}\right)$.

The term $f . a$ in Equation 4 ensures that the atom is centred according to vertical axis at $t_{0}$.

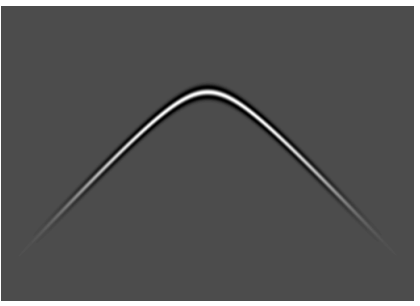

(a) $f=50 ; \mathrm{a}=1.5 ; \sigma=5$

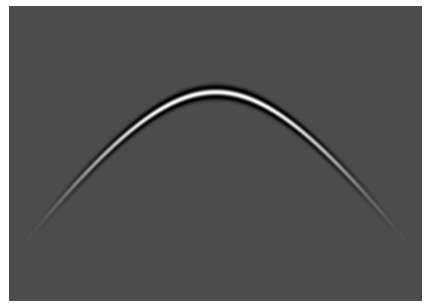

(b) $f=150 ; \mathrm{a}=2 ; \sigma=5$
Figure 1: Atom examples with different parameters
The atoms have the particularity to be absolutely integrable $\left(\mathrm{L}_{1}\left(\mathbb{R}^{2}\right)\right.$ ) and square integrable $\left(\mathrm{L}_{2}\left(\mathbb{R}^{2}\right)\right)$ for $\alpha>0$. This property is ensured by the attenuation term $e^{-\alpha \cdot A(x, t)}$.

Because the hyperbola shape mainly depends on the parameter $a$, in practice to keep a reasonable dictionary size, $\alpha, f$ and $\sigma$ are set such that only one shape parameter and two position parameters remain.

For $c_{k}$, the coefficient maps related to an centred atom $h_{k}$ of a dictionary composed of $K$ atoms, the B-scan $Y$ can be defined by:

$$
\begin{gathered}
Y(x, t)=\sum_{k=1}^{K} \sum_{u, v} c_{k}(u, v) \cdot h_{k}(u-x, v-t) \\
Y=\sum_{k=1}^{K} c_{k} \star h_{k}
\end{gathered}
$$

The coefficients $c_{k}$ have to be computed and analysed in order to extract the useful information.

\section{Proposed Method}

\section{A. Sparse Coding issue with B-scans}

To use the atom centred dictionary and to work faster, convolutional sparse coding is applied to compute the coefficients $c_{k}$ and to ensure a satisfying reconstruction of B-scan $Y$. As the sparse coding, this method constraints the solution (i.e $c_{k}$ ) to be sparse. In theory, the sparsity is applied thanks to the $l_{0}$-norm such that $\|x\|_{0} \triangleq \operatorname{Card}\left\{i\right.$, s.t. $\left.x_{i} \neq 0\right\}$ (with the cardinality of the set $x$ denoted $\operatorname{Car} d\{x\}$ ). However the function minimization with $l_{0}$ constraint is not convex. To overcome this problem the $l_{1}$-norm is preferred; $\|x\|_{1} \triangleq \sum_{k}\left|x_{k}\right|$

In a physical point of view this constraint makes sense because we attend to find scarce reflections on point objects in a Bscan. The formulation of the function minimization is given by :

$$
\underset{\left\{c_{1}, \ldots, c_{K}\right\}}{\arg \min }\left\|\sum_{k}^{K} h_{k} \star c_{k}-Y\right\|_{2}^{2}+\varepsilon \sum_{k}^{K}\left\|c_{k}\right\|_{1}
$$

$\varepsilon$ controls the sparsity level. Higher $\varepsilon$ value, sparser are the coefficients.

To solve this problem several methods have been proposed. In [11], the solution is computed by using the Alternating Method of Multipliers (ADMM) algorithm. ADMM [12] solves successively subproblems to minimize the augmented Lagrangian function. In [13] and [14], an adapted version of respectively FISTA and Matching Pursuit algorithm are detailed with convolution operator.

For a satisfying reconstruction, an important amount of coefficients are necessary to reconstruct some part of a B-scan, especially the direct wave and the background namely the clutter $\mathrm{cl}$. It is mostly represented by a horizontal structure.

This remark is illustrated in Figure 2 where ADMM algorithm has been computed for a simulated B-scan with a perfect clutter and three hyperbolas. Figure 2 (b) shows the coefficient repartition for a single $c_{k}$. A lot of coefficients are needed to decompose clutter with the hyperbola dictionary. Another example is also presented in Figure 3 with a real B-scan. 


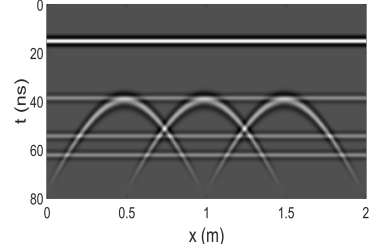

(a) Simulated example

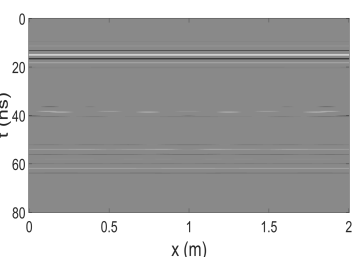

(b) Coefficient repartition
Figure 2: Simulated data : Coefficient repartition for $c_{k}$

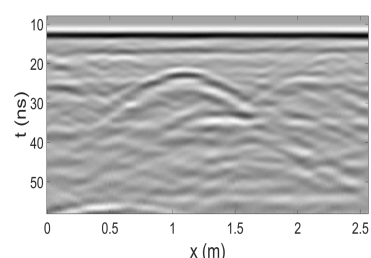

(a) B-scan

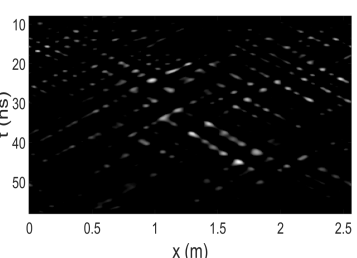

(b) Coefficient repartition
Figure 3: Real data : Coefficient repartition for $c_{k}$

\section{B. Blind Source Separation}

In order to increase the sparsity of the coefficients $c_{k}$, a second constraint is imposed to our minimization problem. Because of the horizontal shape of the clutter and its high correlation with nearby A-scans, we assume that the clutter is a low rank matrix. Then our B-scan is composed of a low rank matrix $L$ (of the same dimension as $Y$ ) and few elements of the hyperbola dictionary. Similarly to the previously exposed method, ADMM algorithm is applied. The objective function is given by:

$$
\begin{array}{rc}
\text { minimize } & \|L\|_{*}+\varepsilon\|S\|_{1} \\
\text { s.t. } & Y=\sum_{k} h_{k} \star c_{k}+L \\
& S=C
\end{array}
$$

with $C=\left(c_{1}, c_{2}, \ldots, c_{K}\right), S$ is a matrix of the same dimension as $C, H=\left(h_{1}, h_{2}, \ldots, h_{K}\right)$. The nuclear norm of the matrix $\mathrm{X}:\|X\|_{*} \triangleq \sum_{i} \sigma_{i}$ i.e the sum of the singular value $\sigma_{i}$ of the matrix $X$. The weighting constant $\varepsilon$ controls the sparsity level of the coefficients $S$ and intrinsically the rank of the matrix $L$. When $\varepsilon$ decreases then we are in the similar case of a convolutional sparse coding with ADMM.

The 2D discrete Fourier transform operator of $X$ and its inverse are given by $\hat{X}=\mathcal{F}(X)$ and $X=\mathcal{F}^{-1}(\hat{X})$.

In order to lighten the next formulations, the operators $\mathcal{P}$, $\hat{\mathcal{P}}$ and $\hat{\mathcal{P}}^{H}$ respectively the reconstruction in time domain, in Fourier domain and the coefficient computation in Fourier domain are defined such that $\mathcal{P} C=\sum_{k} h_{k} \star c_{k}$, $\mathcal{P} C \simeq \mathcal{F}^{-1}(\hat{\mathcal{P}} \hat{C}), \hat{\mathcal{P}} \hat{C}=\sum_{k} \hat{h}_{k} \circ \hat{c}_{k}$ and $\hat{\mathcal{P}}^{H} \hat{Y}=\hat{H}^{H} \circ \hat{Y}$ ( $\circ$ is the Hadamard product and $x^{H}$ is the conjugate of $x$ ). The stopping criteria is defined such that $\operatorname{Derr}^{i+1}=e r r_{Y}^{i+1}-$ $\operatorname{err}_{Y}^{i}<\delta$ with $\operatorname{err}_{Y}^{i}=\mathcal{P} S^{i}+L^{i}-Y$

The subsequent part describes the subproblem solutions of the ADMM algorithm.
Firstly, the augmented Lagrangian is defined by :

$$
\begin{aligned}
& \mathcal{L}_{\rho}\left(L, S, C, \lambda_{y}, \lambda_{s}\right)=\|L\|_{*}+\varepsilon\|S\|_{1}+ \\
& \left\langle\lambda_{y}, \mathcal{P} C+L-Y\right\rangle+\frac{\rho_{y}}{2}\|\mathcal{P} C+L-Y\|_{2}^{2}+ \\
& \left\langle\lambda_{s}, S-C\right\rangle+\frac{\rho_{s}}{2}\|S-C\|_{2}^{2}
\end{aligned}
$$

with $\rho=\left\{\rho_{y}, \rho_{s}\right\}$ the Lagrangian constants.

A solution $\tilde{C}$ is found by minimization of the augmented Lagrangian with the primal and dual variables $\left\{L, S, \lambda_{y}, \lambda_{s}\right\}$ set. In order to solve this problem without the convolution operator we work in the Fourier domain.

$$
\begin{aligned}
& \tilde{C}= \underset{C}{\arg \min } \mathcal{L}_{\rho}\left(C ; L, S, \lambda_{y}, \lambda_{s}\right) \\
&= \mathcal{F}^{-1}\left(\underset{\hat{C}}{\arg \min }\left\langle\hat{\lambda}_{y}, \hat{\mathcal{P}} \hat{C}+\hat{L}-\hat{Y}\right\rangle+\frac{\rho_{y}}{2}\|\hat{\mathcal{P}} \hat{C}+\hat{L}-\hat{Y}\|_{2}^{2}\right. \\
&\left.\quad+\left\langle\hat{\lambda}_{s}, \hat{S}-\hat{C}\right\rangle+\frac{\rho_{s}}{2}\|\hat{S}-\hat{C}\|_{2}^{2}\right) \\
&\left(\rho_{y} \hat{\mathcal{P}} \hat{H}^{H}+\rho_{s} \mathcal{I}\right) \hat{C}=\hat{\mathcal{P}}^{H}\left(\rho_{y}(\hat{Y}-\hat{L})-\hat{\lambda}_{y}\right)+\hat{\lambda}_{s}+\rho_{s} \hat{S}
\end{aligned}
$$

As in [11], the Sherman-Morrison formula is applied to the one-rank inversion problem of Equation 11 :

$$
\left(A+U V^{T}\right)^{-1}=A^{-1}-\frac{A^{-1} U V^{T} A^{-1}}{1+V^{T} A^{-1} U}
$$

Let $\hat{B}$ the second member in Equation 11 :

$\hat{B}=\hat{\mathcal{P}}^{H}\left(\rho_{y}(\hat{Y}-\hat{L})-\hat{\lambda}_{y}\right)+\hat{\lambda}_{s}+\rho_{s} \hat{S}$

$$
\tilde{C}=\mathcal{F}^{-1}\left(\frac{1}{\rho_{s}}\left(\hat{B}-\frac{\rho_{y} \hat{\mathcal{P}}^{H}(\hat{\mathcal{P}} \hat{B})}{\rho_{s}+\rho_{y} \hat{\mathcal{P}} \hat{H}^{H}}\right)\right)
$$

From the augmented Lagrangian, a solution $\tilde{S}$ is defined as :

$$
\begin{aligned}
\tilde{S} & =\underset{S}{\arg \min } \mathcal{L}_{\rho}\left(S ; L, C, \lambda_{y}, \lambda_{s}\right) \\
& =\varepsilon\|S\|_{1}+\left\langle\lambda_{s}, S-C\right\rangle+\frac{\rho_{s}}{2}\|S-C\|_{2}^{2} \\
& =\operatorname{prox}_{\varepsilon \rho_{s}^{-1}\|\cdot\|_{1}}\left(C-\frac{\lambda_{s}}{\rho_{s}}\right)
\end{aligned}
$$

The solution $S$ has the convenient form to be expressed from a proximal operator. Let $\mathcal{T}_{\tau}$ denote the shrinkage operator given by $\mathcal{T}_{\tau}(x)=\operatorname{sgn}(x) \max (|x|-\tau, 0)$

$$
\tilde{S}=\mathcal{T}_{\varepsilon / \rho_{s}}\left(C-\frac{\lambda_{s}}{\rho_{s}}\right)
$$

An analogue way is used to find an optimal solution for $L$.

$$
\begin{aligned}
\tilde{L} & =\underset{L}{\arg \min } \mathcal{L}_{\rho}\left(L ; S, C, \lambda_{y}, \lambda_{s}\right) \\
& =\|L\|_{*}+\left\langle\lambda_{y}, \mathcal{P} C+L-Y\right\rangle+\frac{\rho_{y}}{2}\|\mathcal{P} C+L-Y\|_{2}^{2} \\
& =\operatorname{prox}_{\rho_{y}^{-1}\|\cdot\|_{*}}\left(Y-\mathcal{P} C-\frac{\lambda_{y}}{\rho_{y}}\right)
\end{aligned}
$$

Here, the singular value thresholding operator $\mathcal{S} \mathcal{V} \mathcal{T}$ is used [16]. $\mathcal{S} \mathcal{V} \mathcal{T}_{\tau}(X)=U \mathcal{T}_{\tau}(\Sigma) V^{T}$, with $X=U \Sigma V^{T}$

$$
\tilde{L}=\mathcal{S} \mathcal{V} \mathcal{T}_{1 / \rho_{y}}\left(Y-\mathcal{P} C-\frac{\lambda_{y}}{\rho_{y}}\right)
$$


Finally, the Lagrange multipliers are updated:

$$
\begin{aligned}
& \lambda_{y}^{i+1}=\lambda_{y}^{i}+\mathcal{P} C^{i+1}+L^{i+1}-Y \\
& \lambda_{s}^{i+1}=\lambda_{s}^{i}+S^{i+1}-C^{i+1}
\end{aligned}
$$

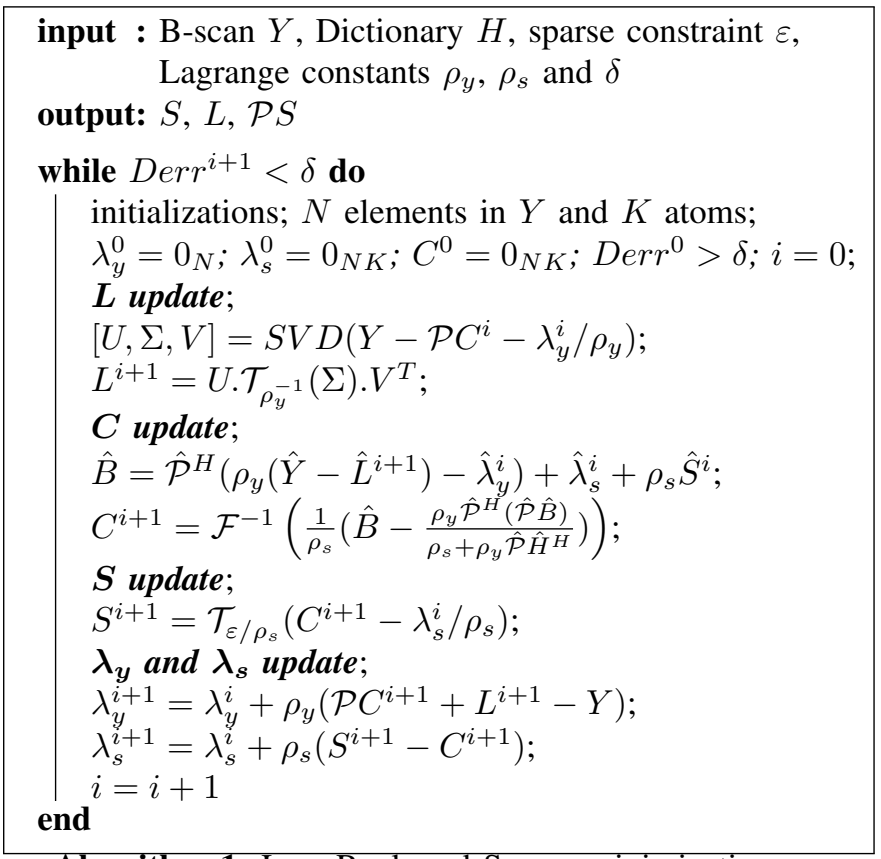

Algorithm 1: Low Rank and Sparse minimization

\section{RESUlTs}

In order to asses the performances of our method, the results on simulated and real B-scans are presented.

Firstly we applied our method on simulated B-scans composed of a perfect clutter and several hyperbolas where their positions have been randomly chosen. The performances for different numbers of hyperbolas are presented in Table I. The number of coefficients $\left(\|\alpha\|_{0}\right)$ used for the reconstruction are close to the hyperbola number $\left(N_{\text {hyp }}\right)$ with a low reconstruction error on $L$ and $Y$. Moreover the additional coefficients are located in a close area to the solution.

\begin{tabular}{|c|c|c|c|c|c|}
\hline$N_{\text {hyp }}$ & $\varepsilon$ & $\|\alpha\|_{1}$ & $\|\alpha\|_{0}$ & $\frac{\left\|\tilde{L}-L_{0}\right\|_{F}}{\left\|L_{0}\right\|_{F}}$ & $\frac{\|\tilde{Y}-Y\|_{F}}{\|Y\|_{F}}$ \\
\hline 3 & 0.9 & 0.659 & 9 & 0.005 & 0.007 \\
\hline 10 & 0.7 & 1.877 & 10 & 0.001 & 0.010 \\
\hline 20 & 0.5 & 3.166 & 26 & 0.002 & 0.009 \\
\hline 50 & 0.4 & 5.7 & 69 & 0.008 & 0.0133 \\
\hline
\end{tabular}

Table I: Results of the reconstruction error, the sparsity level and error on the low rank matrix $L$ and B-scan $Y$ for different values of $\varepsilon$ on simulated $\mathrm{B}$-scans

Figure 6 shows the relation between the sparsity level of $S$ and the rank of $L$ according to the weighting constant $\varepsilon$. When the sparsity level is far from the "real" sparsity, the algorithm does not correctly recover the matrix $L$.

Figure 4 shows the results for a simulated B-scan. The dictionary coupled with the low rank matrix constraint allow an efficient clutter removal without impacting the hyperbolas.
Then our method has been compared with the convolutional FISTA [13] and ADMM with $l_{1}$ constraint [11] on real data. An inverse operator has also been computed with a Conjugate Gradient algorithm [15]. Thus a dictionary is used to compute the coefficients and a second one for the reconstruction. The last method directly offers a coefficient computation and a reconstruction without any iteration. However the coefficients are not sparse.

The results are presented in Table II for different weighting constants. The red rows indicate the large reconstruction errors. The methods GC, FISTA, ADMM $l_{1}$ only present a low reconstruction error when an important amount of coefficients are used (i.e with a low $\varepsilon$ ). In comparison, our method shows a sparser ( $\|\alpha\|_{1}$ and $\|\alpha\|_{0} / N$ with $N$ coefficients) and better reconstruction (PSNR and $\|\tilde{Y}-Y\|_{F} /\|Y\|_{F}$ ). However it needs more iterations $\left(N_{\text {iter }}\right)$ to converge. Figure 5 shows the blind source separation of a real B-scan. The useful signal has been decomposed with few coefficients and has been well separated from the clutter.

\begin{tabular}{|c||c|c|c|c|c|c|}
\hline Algorithm & $\varepsilon$ & PSNR & $\frac{\|\tilde{Y}-Y\|_{F}}{\|Y\|_{F}}$ & $\|\alpha\|_{1}$ & $\frac{\|\alpha\|_{0}}{N}(\%)$ & $N_{\text {iter }}$ \\
\hline CG & $\times$ & 31.4 & 0.0409 & $\times$ & $\times$ & $\times$ \\
\hline \multirow{2}{*}{ FISTA } & 0.0001 & 25.2 & 0.0822 & 127.0585 & 100 & 210 \\
& 0.001 & 14.3 & 0.2888 & 89.4755 & 99.8 & 201 \\
& 0.005 & 3.8 & 0.9667 & 2.0845 & 2.4 & 271 \\
\hline \multirow{3}{*}{ ADMM $\mathbf{l}_{\mathbf{1}}$} & 0.0001 & 39.3 & 0.0162 & 136.4 & 100 & 10 \\
& 0.001 & 21.6 & 0.1241 & 103.5 & 42.5 & 47 \\
& 0.005 & 8.5 & 0.5607 & 47.3 & 4.0 & 177 \\
\hline \multirow{3}{*}{ ADMM $\mathbf{l}_{\mathbf{1}}+\mathbf{l}_{*}$} & 0.01 & 50.6 & 0.0044 & 5.0960 & 2.44 & 136 \\
& 0.1 & 42.2 & 0.0116 & 1.2667 & 0.14 & 485 \\
& 0.2 & 39.3 & 0.0161 & 0.8676 & 0.06 & 783 \\
\hline
\end{tabular}

Table II: Results of the reconstruction error and the sparsity level for different methods on real data

The proposed method could be used to enhance hyperbola signature or to automatically detect hyperbolas in B-scan with a wise coefficient analysis. Indeed the low number of required coefficients for the reconstruction will simplify the coefficient selection from physical restriction for example. Figure 7 illustrates the reconstruction from the ten highest coefficients computed from the B-scan example in Figure 3 (a). We notice that these coefficients are mostly associated to the main hyperbola from the B-scan.

\section{CONClusion}

In this work, we have presented an hyperbola dictionary for GPR data application. This dictionary is used in a blind source separation scheme to distinguish the clutter and a sparse representation of the useful signal. The sparsity will simplify the coefficient analysis in a further step for the automatic hyperbola detection or hyperbola signature enhancement. We have shown that our method allows a reconstruction with sparser coefficients of the useful signal than presented methods and a clutter removal without impacting the hyperbolas. However this method needs more iterations to converge and the estimation of the clutter can be unsatisfactory for a wrong estimation of $\varepsilon$. In future work, an automatic estimation of $\varepsilon$ will be studied and an efficient way to exploit the sparse coefficients. 


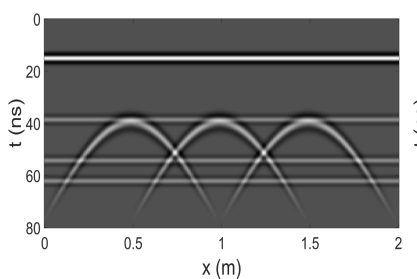

(a) Simulated B-scan

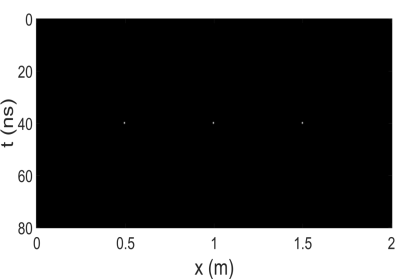

(b) Coefficient sum

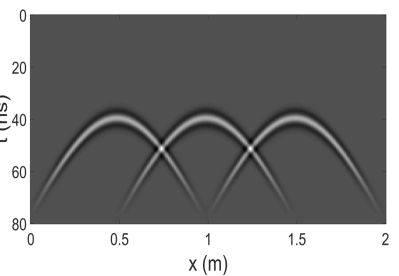

(c) $\mathcal{P} S$

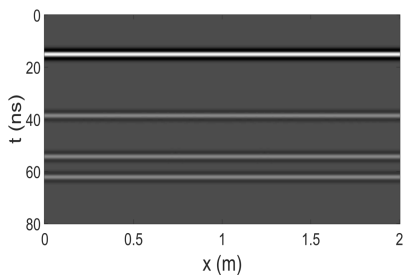

(d) $L$

Figure 4: Blind source separation results for a simulated B-scan; (b) Sum of coefficients $c_{k}$ at each position $(x, t)$; (c) Reconstruction from the coefficients $S$ and the dictionary $\mathcal{P} S$; (d) The low rank matrix $L$

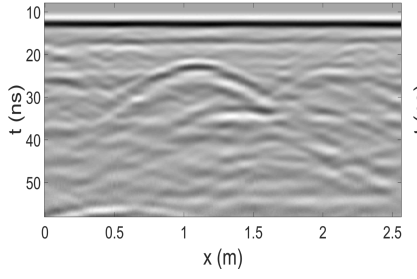

(a) Real B-scan

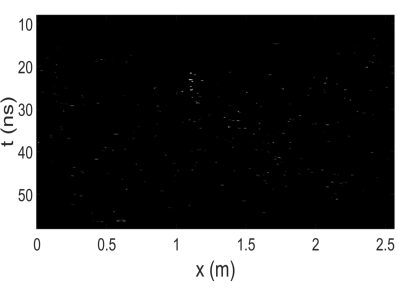

(b) Coefficient sum

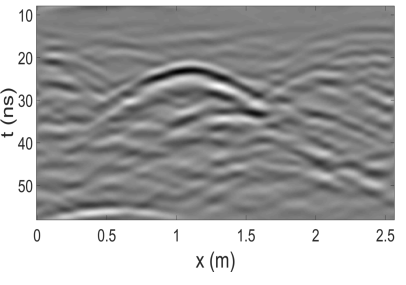

(c) $\mathcal{P} S$

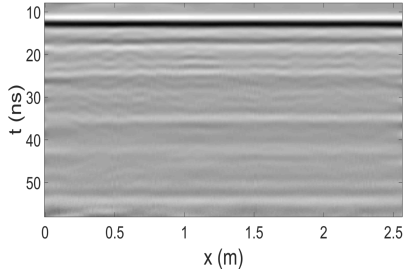

(d) $L$

Figure 5: Blind source separation results for a real B-scan; (b) Sum of coefficients $c_{k}$ at each position $(x, t)$; (c) Reconstruction from the coefficients $S$ and the dictionary $\mathcal{P} S$; (d) The low rank matrix $L$

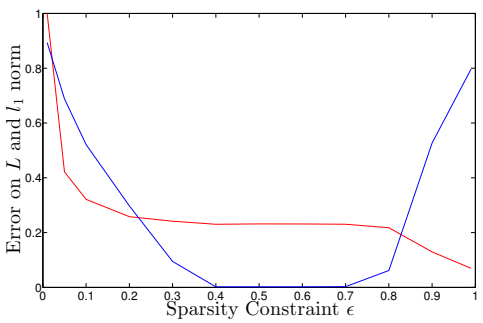

Figure 6: According to the weighting constant $\varepsilon$; in blue : Evolution of the coefficient sparsity level of $S$ (normalized to $1)$; in red : the reconstruction error on the low rank matrix $L$

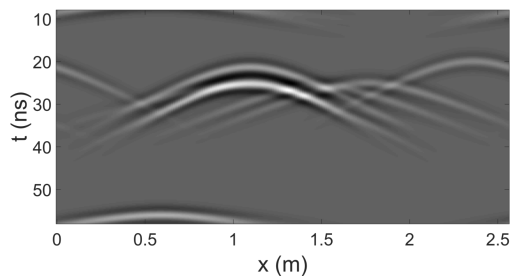

Figure 7: Reconstruction with the ten highest coefficients

\section{ACKNOWLEDGEMENT}

The authors would like to thank BPI France and Région Îlede-France which have supported this work within the G4M project (http://g4m.fr).

\section{REFERENCES}

[1] H. Jol, Ground penetrating radar, theory and applications. Elsevier, 2009.

[2] I. J. Gupta, A. van der Merwe, and C.-C. Chen, "Extraction of complex resonances associated with buried targets," pp. 1022-1032, 1998
[3] D. Potin, E. Duflos, and P. Vanheeghe, "Landmines Ground-Penetrating Radar Signal Enhancement by Digital Filtering," IEEE Transactions on Geoscience and Remote Sensing, vol. 44, no. 9, pp. 2393- 2406, 2006.

[4] G. Terrasse, J. M. Nicolas, E. Trouvé, and E. Drouet, "Application of the curvelet transform for pipe detection in gpr images," in IGARSS, Milano, Italy, Jul. 2015.

[5] R. Zhang and T. J. Ulrych, "Physical wavelet frame denoising," Geophysics, vol. 68, no. 1, pp. 225-231, 2003.

[6] M. Elad and M. Aharon, "Image denoising via learned dictionaries and sparse representation," in Computer Vision and Pattern Recognition, 2006 IEEE Computer Society Conference on, vol. 1, June 2006, pp. 895-900.

[7] D.-S. Pham and S. Venkatesh, "Joint learning and dictionary construction for pattern recognition," in Computer Vision and Pattern Recognition, 2008. CVPR 2008. IEEE Conference on, June 2008, pp. 1-8.

[8] E. J. Candès, X. Li, Y. Ma, and J. Wright, "Robust principal component analysis?" J. ACM, vol. 58, no. 3, pp. 11:1-11:37, Jun. 2011.

[9] S. Mallat and Z. Zhang, "Matching pursuit with time-frequency dictionaries," IEEE Transactions on Signal Processing, vol. 41, pp. 3397$3415,1993$.

[10] A. Beck and M. Teboulle, "A fast iterative shrinkage-thresholding algorithm for linear inverse problems," SIAM J. Img. Sci., vol. 2, no. 1, pp. 183-202, Mar. 2009.

[11] B. Wohlberg, "Efficient convolutional sparse coding," in Acoustics, Speech and Signal Processing (ICASSP), 2014 IEEE International Conference on, May 2014, pp. 7173-7177.

[12] S. Boyd, N. Parikh, E. Chu, B. Peleato, and J. Eckstein, "Distributed optimization and statistical learning via the alternating direction method of multipliers," Found. Trends Mach. Learn., vol. 3, no. 1, pp. 1-122, Jan. 2011.

[13] R. Chalasani, J. Principe, and N. Ramakrishnan, "A fast proximal method for convolutional sparse coding," in Neural Networks (IJCNN), The 2013 International Joint Conference on, Aug 2013, pp. 1-5.

[14] A. Szlam, K. Kavukcuoglu, and Y. LeCun, "Convolutional matching pursuit and dictionary training," CoRR, vol. abs/1010.0422, 2010.

[15] K. Grochenig, "Acceleration of the frame algorithm," Signal Processing, IEEE Transactions on, vol. 41, no. 12, pp. 3331-3340, Dec 1993.

[16] J.-F. Cai, E. J. Candès, and Z. Shen, "A singular value thresholding algorithm for matrix completion," SIAM J. on Optimization, vol. 20 , no. 4, pp. 1956-1982, Mar. 2010. 